\title{
ELLIPTIC CURVES IN TWO-DIMENSIONAL ABELIAN VARIETIES AND THE ALGEBRAIC INDEPENDENCE OF CERTAIN NUMBERS
}

Robert Tubbs

I. Introduction. A fruitful line of study in transcendental number theory has been an investigation into the number of algebraically independent values which belong to some prescribed set. The points under consideration are usually associated with the ordinary exponential function, or, more recently, with a Weierstrass elliptic function. In this paper we find some conditions which imply that a nontrivial one-parameter subgroup of a two-dimensional abelian variety is contained in an elliptic curve. From this we deduce several consequences concerning the algebraic independence (or transcendence) of certain values.

THEOREM. Let $A$ be a two-dimensional abelian variety defined over $\overline{\mathbf{Q}}$ and $\phi: \mathbf{C} \rightarrow A(\mathbf{C})$ a nontrivial analytic homomorphism which is defined over some subfield $K$ of $\mathbf{C}$ (i.e., $\phi^{\prime}(0) \in J_{A}(K)$ where $J_{A}$ denotes the tangent space of $A$ at its identity element $)$. Suppose $y_{0}, y_{1}, y_{2}, y_{3}$ are linearly independent complex numbers with $\phi\left(y_{i}\right) \in A(K)(0 \leq i \leq 3)$ such that either

(a) $\phi\left(y_{0}\right) \in A(\overline{\mathbf{Q}})_{\text {tors }}$, or

(b) $\phi\left(y_{0}\right) \in A(\overline{\mathbf{Q}})$ and $y_{0}, y_{1}, y_{2}, y_{3} \in K$.

Then trans $\operatorname{deg}_{\mathbf{Q}} K \leq 1$ implies that $\overline{\phi(\mathbf{C})}$ is an elliptic curve.

COROLLARY 1 (Elliptic analogue to the Brownawell-Waldschmidt theorem; [3], [10]). Let $\odot(z)$ be a Weierstrass elliptic function with algebraic invariants and let $\mathcal{O}$ denote the ring of multiplications of $\mathcal{} \odot$. Suppose that $\left\{u_{1}, u_{2}\right\}$ are $\mathcal{O}$ linearly independent and $\left\{v_{1}, \ldots, v_{4}\right\}$ are $\mathbf{Z}$-linearly independent sets of complex

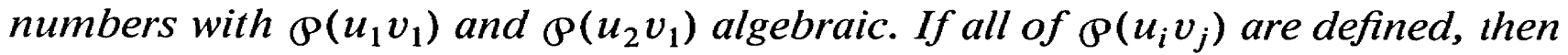
at least two of

$$
u_{i}, v_{j}, \mathcal{P}\left(u_{i} v_{j}\right) \quad(1 \leq i \leq 2,1 \leq j \leq 4)
$$

are algebraically independent.

Proof. Let $E$ be the elliptic curve associated with $\odot(z)$, put $A=E \times E$ and

$$
\phi(z)=\left(1, \odot\left(u_{1} z\right), \Phi^{\prime}\left(u_{1} z\right), 1, \odot\left(u_{2} z\right), \Phi^{\prime}\left(u_{2} z\right)\right) .
$$

$\phi(C)$ is Zariski dense in $A$ since the $\mathcal{O}$-linear independence of $u_{1}, u_{2}$ implies that $\mathcal{P}\left(u_{1} z\right)$ and $\mathcal{P}\left(u_{2} z\right)$ are algebraically independent [4].

Put $K=\overline{\mathbf{Q}}\left(u_{i}, v_{j}, \propto\left(u_{i} v_{j}\right)\right), 1 \leq i \leq 2,1 \leq j \leq 4$. Then $\phi^{\prime}(0) \in J_{A}(K), \phi\left(v_{1}\right) \in$ $A(\overline{\mathbf{Q}})$ and case (b) of the Theorem implies trans $\operatorname{deg}_{\mathbf{Q}} K \geq 2$.

Received February 4, 1985. Revision received March 16, 1986.

Research supported in part by the National Science Foundation Grant MCS-8108814(A04).

Michigan Math. J. 34 (1987). 
COROLLARY 2. Suppose that $\mathcal{P}(z)$ has algebraic invariants. For any $u \in \mathbf{C}$ at least one of

$$
\mathcal{P}(u), \mathcal{P}\left(u^{2}\right), \mathcal{P}\left(u^{3}\right), \mathcal{P}\left(u^{4}\right), \mathcal{P}\left(u^{5}\right)
$$

is transcendental (whenever they are all defined).

Proof. If $u$ is algebraic, $u \neq 0$, then it is a basic result of Schneider's that $\rho(u)$ is transcendental. When $u$ is transcendental, apply Corollary 1 with $u_{1}=u, u_{2}=$ $u^{2}, v_{1}=1, v_{2}=u, v_{3}=u^{2}, v_{4}=u^{3}$. Since $u_{i}$ and $v_{j}(1 \leq i \leq 2,1 \leq j \leq 4)$ are algebraically dependent, Corollary 2 follows.

II. Preliminary results. The proof of the Theorem relies on some recent joint work of Masser and Wüstholz. However, the following generalization of a result of Gelfond's, due to Brownawell, is central.

LEMMA 1. Let $a>1$ and suppose that $\left(\delta_{t}\right)_{t \in \mathrm{N}}$ and $\left(\gamma_{t}\right)_{t \in \mathrm{N}}$ are positive, strictly monotonic, unbounded sequences such that for each $t \in \mathbf{N}$

$$
\delta_{t+1} \leq a \delta_{t}, \quad \gamma_{t+1} \leq a \gamma_{t} .
$$

Let $\theta \in \mathbf{C}$. If there exists a sequence of nonzero integral polynomials $P_{t}(X)$ such that for each $t \in \mathbf{N}$

and

$$
\operatorname{deg} P_{t} \leq \delta_{t}, \quad \log \text { ht } P_{t} \leq \gamma_{t}
$$

$$
\log \left|P_{t}(\theta)\right|<-(2 a+1) \delta_{t}\left(\delta_{t}+\gamma_{t}\right) .
$$

then $\theta$ is algebraic and $P_{t}(\theta)=0$ for each $t \in \mathbf{N}$.

Proof. See [2, Theorem 1].

Masser and Wüstholz have provided estimates for the number of zeros (with respect to a certain measure of multiplicity) of a polynomial on a finite subset of a quasi-projective group variety $G$. Suppose that the dimension of $G$ is $d$ and $\Gamma$ is a finitely generated subgroup of $G$ of rank $\ell>0$. For each integer $r, 1 \leq r \leq d$, define an integer $p_{r}$ as the minimum corank of any subgroup $\Gamma$ which lies in some algebraic subgroup of $G$ of codimension $r$. If $G$ has no codimension $r$ algebraic subgroups put $p_{r}=\ell$. When $\Gamma=\mathbf{Z}_{\gamma_{1}}+\cdots+\mathbf{Z}_{\gamma_{\ell}}$ we also use the notation

$$
\Gamma(S)=\mathbf{Z}(S) \gamma_{1}+\cdots+\mathbf{Z}(S) \gamma_{\ell},
$$

where $\mathbf{Z}(S)=\{n: 0 \leq n<S\}$.

Let $\Psi: G \hookrightarrow \mathbf{P}_{N}$ be an embedding as in [7]. If $J_{G}$ denotes the tangent space of $G$ at its identity element with coordinates $z_{1}, \ldots, z_{d}$, then $\exp _{G}: \mathfrak{J}_{G} \rightarrow G \subseteq \mathbf{P}_{N}$ is given by holomorphic functions $f_{0}, \ldots, f_{N}$. When $\phi: \mathbf{C} \rightarrow G(\mathbf{C})$ is a nontrivial analytic homomorphism there exists an injective linear map $\mathscr{L}: \mathbf{C} \rightarrow \mathfrak{J}_{G}$ such that $\phi: \mathbf{C} \rightarrow G(\mathbf{C}) \subseteq \mathbf{P}_{N}$ is given by $\phi=\exp _{G} \circ \mathcal{L}$. The condition $\phi^{\prime}(0) \in \mathfrak{J}_{G}(K)$ insures that $\mathscr{L}$ is defined over $K$.

Now suppose that $G=G_{1} \times \cdots \times G_{k}$ is a product of group varieties $G_{1}, \ldots, G_{k}$ with each $G_{i}$ embedded in $\mathbf{P}_{N_{i}}(1 \leq i \leq k)$. We define the order of vanishing of $P$ 
at $g \in G$ along $\phi$ : For each $i, 1 \leq i \leq k$, choose $z_{i} \in J_{G_{i}}$ with $\exp _{G_{i}}\left(z_{i}\right)=\pi_{i}(g)$, $\pi_{i}: G \rightarrow G_{i}$ being the projection map. Then if $\exp _{G_{i}}(z)$ is given by the holomorphic functions $\left(f_{0}^{(i)}(z), \ldots, f_{N_{i}}^{(i)}(z)\right)$ choose $j_{i}=j(i, g)$ with $f_{j_{i}}^{(i)}\left(z_{i}\right) \neq 0$. Let

$$
\chi(\zeta)=P\left(\frac{f_{0}^{(1)}}{f_{j_{1}}^{(1)}}\left(z_{1}+\mathfrak{L}_{1}(\zeta)\right), \ldots, \frac{f_{N_{k}}^{(k)}}{f_{j_{k}}^{(k)}}\left(z_{k}+\mathfrak{L}_{k}(\zeta)\right)\right),
$$

where $\pi_{i} \circ \phi(z)=\exp _{G_{i}} \circ \mathfrak{L}_{i}$, with $\mathfrak{L}_{i}: \mathbf{C} \rightarrow \mathfrak{J}_{G_{i}}(\mathbf{C})$ linear. Define the order of vanishing of $P$ at $g$ along $\phi$ by:

$$
\operatorname{ord}_{g} P=\left\{\begin{array}{l}
\infty, \text { if } \chi(\zeta)=0, \\
\left\{\max T:\left.\left(\frac{d}{d \zeta}\right)^{t} \chi(\zeta)\right|_{\zeta=0}=0, \text { for all } t<T\right\}, \text { otherwise; }
\end{array}\right.
$$

$\operatorname{ord}_{g} P$ is independent of our choices $z_{i}$ and $f_{j_{i}}^{(i)}(z)$.

Suppose that $G_{1}, \ldots, G_{k}$ have dimensions $d_{1}, \ldots, d_{k}$ respectively and that $\Gamma=$ $\Gamma_{1}+\cdots+\Gamma_{h}$ is a sum of finitely generated subgroups $\Gamma_{1}, \ldots, \Gamma_{h}$ of $G$ of ranks $\ell_{1}, \ldots, \ell_{n}$ respectively. Let $\phi: \mathbf{C} \rightarrow G(\mathbf{C})$ be an analytic homomorphism of $G$. In this context Masser and Wüstholz have provided the following result.

LEMMA 2. There exists a constant $C_{G}$ depending only on $G_{1}, \ldots, G_{k}$ and their embeddings in $\mathbf{P}_{N_{i}}$ with the following property. Suppose for some real numbers $S_{1} \geq 0, \ldots, S_{h} \geq 0, D_{1} \geq 0, \ldots, D_{k} \geq 0$, and $T \geq 0$ there exists a multihomogeneous polynomial $P$ of multidegree $\left(D_{1}, \ldots, D_{k}\right)$ which vanishes along $\phi$ to order at least $T$ on $\Gamma_{1}\left(S_{1}\right)+\cdots+\Gamma_{h}\left(S_{h}\right)$. For each $r, 1 \leq r \leq d=d_{1}+\cdots+d_{k}$, let $\Sigma_{r}$ equal the minimum of the products $p_{r}$ at a time of the numbers $S_{1}, \ldots, S_{1}\left(\ell_{1}\right.$ times $), \ldots$, $S_{h}, \ldots, S_{h}\left(\ell_{h}\right.$ times $)$ and $\Delta_{r}$ equal the maximum of the products $r$ at a time of the numbers $D_{1}, \ldots, D_{1}\left(d_{1}\right.$ times $), \ldots, D_{k}, \ldots, D_{k}\left(d_{k}\right.$ times $)$. If

and

$$
T \Sigma_{r} \geq C_{G} d^{p_{r} \Delta_{r}} \quad(1 \leq r \leq d)
$$

$$
E \Sigma_{r} \geq C_{G} d^{p_{r} \Delta_{r}} \quad(1 \leq r<d),
$$

where $E=\min \left(D_{1}, \ldots, D_{k}\right)$, then $P$ vanishes on all of $\gamma+\phi$ for some $\gamma \in \Gamma$.

Proof. See [6].

III. Proof of the Theorem. If $K \subseteq \overline{\mathbf{Q}}$ then the Theorem follows from Théorème 3.1.1 of [9]; hence we assume that the transcendence degree of $K$ over $\mathbf{Q}$ is 1 . Then there exists a transcendental number $\theta$, and a complex number $\theta_{1}$, integral over $\mathbf{Z}[\theta]$ of degree $n$, where $K=\mathbf{Q}\left[\theta, \theta_{1}\right]$. In this situation let $\mathcal{O}_{K}=\mathbf{Z}\left[\theta, \theta_{1}\right]$. For $\alpha \in K^{*}$ there is a representation

$$
\alpha=\frac{\sum_{i=1}^{n} P_{i} \theta_{1}^{i-1}}{P_{0}}
$$

with $P_{0}, \ldots, P_{n}$ coprime polynomials in $\mathbf{Z}[\theta]$. We put

$$
\begin{aligned}
\operatorname{deg}(\alpha) & =\max \left\{\operatorname{deg} P_{0}, \ldots, \operatorname{deg} P_{n}\right\}, \\
\text { ht }(\alpha) & =\max \left\{\text { ht } P_{0}, \ldots, \text { ht } P_{n}\right\} .
\end{aligned}
$$


We first show that the Theorem holds in case (b). We consider case (a) in Section IV below. Assume that $A, \phi, y_{0}, y_{1}, y_{2}, y_{3}$, and $K$ satisfy the hypotheses of the Theorem (with $y_{0}, y_{1}, y_{2}, y_{3}$ all in $K$ ) but not the conclusion. When $\overline{\phi(\mathbf{C})}$ is not an elliptic curve, $\operatorname{dim} \overline{\phi(\mathbf{C})}=2$ and $\phi(\mathbf{C})$ is Zariski dense in $A$.

Let

$$
Y=y_{0} \mathbf{Z}+y_{1} \mathbf{Z}+y_{2} \mathbf{Z}+y_{3} \mathbf{Z}
$$

and for a vector of non-negative reals $S=\left(S_{0}, S_{1}, S_{2}, S_{3}\right)$ put

$$
Y(S)=y_{0} \mathbf{Z}\left(S_{0}\right)+y_{1} \mathbf{Z}\left(S_{1}\right)+y_{2} \mathbf{Z}\left(S_{2}\right)+y_{3} \mathbf{Z}\left(S_{3}\right) \text {. }
$$

For $G=\mathbf{G}_{a} \times A$ the embedding $\Psi: A \rightarrow \mathbf{P}_{N}$ described above may be extended to an embedding $\Psi^{*}: G \rightarrow \mathbf{P}_{1} \times \mathbf{P}_{N}$ defined by $\Psi^{*}(z)=(1, z, \Psi(z))$. Put $\phi=\Psi \circ \phi$ and $\phi^{*}(z)=(1, z, \phi(z)): \mathbf{C} \rightarrow G(\mathbf{C}) \subseteq \mathbf{P}_{1} \times \mathbf{P}_{N}$. A finitely generated subgroup $\Gamma$ of $G$ is then associated with $Y$ by $\Gamma=\phi^{*}(Y)$.

Our next lemma gives estimates for the exponents $p_{r}(\Gamma, G)$, defined above, which suffice for our proofs. To establish these estimates we assume that

$$
\operatorname{rank}_{\mathbf{Z}}(Y \cap \operatorname{ker} \phi)=0 \text {. }
$$

Otherwise we are in the situation of case (a).

LEMMA 3. With $Y, \phi^{*}, \Gamma$, and $G$ as above, let $p_{r}=p_{r}(\Gamma, G)$ for $1 \leq r \leq 3$. Then $p_{1} \geq 2$ and $p_{2}=p_{3}=4$.

Proof. Let $\pi_{1}: G \rightarrow \mathbf{G}_{a}$ denote the projection mapping. Suppose that $\Gamma^{\prime}$ is a finitely generated subgroup of $\Gamma$ of corank $p_{1}$ which is contained in a codimension 1 algebraic subgroup $H$ of $G$. If $\Gamma^{\prime}=0$ then $p_{1}=4$. Otherwise, the connected component of $H$ at the identity element of $G, H^{0}$, has $\pi_{1}\left(H^{0}\right) \neq 0 .\left(\pi_{1}(H)=\right.$ $\bigcup\left(g+\pi_{1}\left(H^{0}\right)\right)$, where the union is over a finite set, which implies that if $\pi_{1}\left(H^{0}\right)=$ 0 then $\pi_{1}(H)$ is a finite subgroup of $\mathbf{G}_{a}$. There are no such finite subgroups.) Hence $\pi_{1}\left(H^{0}\right)=\mathbf{G}_{a}$.

Using Lemma 7 of [6] one sees that $\mathbf{G}_{a}$ and $A$ are "disjoint," so $H^{0}=\mathbf{G}_{a} \times B$ where $B$ is a codimension 1 algebraic subgroup of $A$. Then $G / H^{0}$ is isogeneous to $A / B$ and hence is an elliptic curve $\widetilde{E}$. Choose $Y^{\prime} \subset Y$ such that $\phi^{*}\left(Y^{\prime}\right)=\Gamma^{\prime}$ and $\operatorname{rank}_{\mathbf{Z}}\left(Y^{\prime}\right)=\operatorname{rank}_{\mathbf{Z}}\left(\Gamma^{\prime}\right)$. For the projection map $p: G \rightarrow G / H^{0}$ we have the existence of a linear map $\tilde{\mathscr{L}}: \mathbf{C} \rightarrow \mathbf{C}$ such that $\exp _{\tilde{E}} \circ \tilde{\mathscr{L}}=p \circ \phi^{*}$. If $\tilde{\mathscr{L}}=0$ then $\phi^{*}(\mathbf{C}) \subset$ $H$ and $\overline{\phi(\mathbf{C})} \neq A$. Therefore $\tilde{\mathscr{L}}$ is injective, $\tilde{\mathscr{L}}\left(Y^{\prime}\right) \subset \operatorname{ker}\left(\exp _{\tilde{E}}\right), \operatorname{rank}_{\mathrm{Z}} Y^{\prime} \leq 2$, and $p_{1} \geq 2$.

The arguments that $p_{2}=p_{3}=4$ are a bit simpler. If $\Gamma^{\prime}$ is a finitely generated subgroup of $\Gamma$ of corank $p_{2}$ which is contained in a codimension 2 algebraic subgroup $H$ of $G$, then (as before) $\pi_{1}\left(H^{0}\right)=\mathbf{G}_{a}$. Moreover, $H^{0}=\mathbf{G}_{a} \times T$ where $\operatorname{dim}(T)=0$, and $H^{0}$ connected implies $T=0$. Then by (2)

$$
\operatorname{rank}_{\mathbf{Z}}\left(\Gamma^{\prime}\right) \leq \operatorname{rank}_{\mathbf{Z}}\left(\phi(Y) \cap A_{\text {tors }}\right)=0 .
$$

Therefore $p_{2}=4$.

Finally if $H$ is a codimension 3 algebraic subgroup of $G$, then $H$ is finite. Hence $H \cap \Gamma$ is finite and $p_{3}=4$. 
The above lemma will be used, in conjunction with Lemma 2, to obtain a nonzero value for a certain analytic function whose existence is guaranteed by the following result. Let $\mathcal{G}$ denote the bihomogeneous ideal which defines $G$ in $\mathbf{P}_{1} \times \mathbf{P}_{N}$. The constants $c_{1}, \ldots$ throughout the remainder of this paper depend at most on $G, y_{0}, y_{1}, y_{2}, y_{3}$, and the embedding of $G$ into multiprojective space.

LEMMA 4. Suppose $Y, \phi^{*}, \Gamma$, and $G$ are as above. There exists $D_{1}>0$ such that for every integer $D \geq D_{1}$ there exists a bihomogeneous polynomial

$$
P \in \mathcal{O}_{K}[Y, X] \backslash \mathcal{G}
$$

of bidegree at most $\left(D^{2} \log ^{-1 / 2} D, D\right)$ and with coefficients having

$$
\operatorname{deg} \leq c_{1} D^{2} \log { }^{-1 / 3} D, \quad \log \text { ht } \leq c_{2} D^{2} \log ^{2 / 3} D
$$

such that the function

$$
\Phi(z)=P\left(1, z, \phi_{0}(z), \ldots, \phi_{N}(z)\right)
$$

satisfies

$$
\underset{|z|=r}{\log \max }\left|\Phi^{(t)}(z)\right| \leq-c_{3} D^{4} \log { }^{1 / 2} D
$$

for all $r \leq c_{4} D$ and $t \leq c_{5} D^{3}$.

Proof. For every positive integer $D$ let

$$
\begin{aligned}
& T=\llbracket c^{\prime} D^{2} \log ^{-1 / 3} D \rrbracket, \quad L=\llbracket D^{2} \log ^{-1 / 2} D \rrbracket, \\
& S_{0}=\llbracket D^{1 / 2} \log ^{1 / 3} D \rrbracket, \quad S=\llbracket D^{1 / 2} \log ^{-1 / 6} D \rrbracket,
\end{aligned}
$$

where we take $0<c^{\prime}<1$ below. Also, let $\mathfrak{g}$ denote an indexing set for a maximal set of bihomogeneous monomials

$$
m_{j, i}=Y_{0}^{j_{0}} Y_{1}^{j_{1}} X_{0}^{i_{0}} \cdots X_{N}^{i_{N}}
$$

of bidegree $(L, D)$ which are linearly independent modulo $\mathcal{G}$. Note that card $\mathfrak{I} \geq$ $c_{6} L D^{2}$.

Put

$$
P(Y, X)=\sum_{(j, i) \in \mathcal{S}} p_{j, i} m_{j, i}(Y, X)
$$

with undetermined coefficients $p_{j, i}$ and consider the function $\Phi(z)$ associated with $P$ by (3).

With $S=\left(S_{0}, S, S, S\right)$ we let $\Phi_{y}(z)=\Phi(z+y)$ for each $y \in Y(S)$. Then if $y=$ $s_{0} y_{0}+s_{1} y_{1}+s_{2} y_{2}+s_{3} y_{3}$ there are bihomogeneous polynomials $A_{0}, \ldots, A_{N}$ and $A_{0}^{\prime}, \ldots, A_{N}^{\prime}$ with deg $+\log \mathrm{ht} \leq c_{7}$ such that, for $z$ near 0 ,

$$
\left(A_{0}\left(\phi\left(z+s_{0} y_{0}\right), \phi\left(s_{1} y_{1}+\cdots+s_{3} y_{3}\right)\right), \ldots, A_{N}\left(\phi\left(z+s_{0} y_{0}\right), \phi\left(s_{1} y_{1}+\cdots+s_{3} y_{3}\right)\right)\right)
$$

are projective coordinates of $\phi(z+y)$ and

$$
\left(A_{0}^{\prime}\left(\phi(z), \phi\left(s_{0} y_{0}\right)\right), \ldots, A_{N}^{\prime}\left(\phi(z), \phi\left(s_{0} y_{0}\right)\right)\right)
$$


are projective coordinates of $\phi\left(z+s_{0} y_{0}\right)$. Further, by Lemma 7 of [1], there are trihomogeneous polynomials $F_{0}, \ldots, F_{N}$ with deg $+\log$ ht $\leq c_{8} S^{2}$ such that

$$
\left(\ldots, F_{i}\left(\phi\left(y_{1}\right), \phi\left(y_{2}\right), \phi\left(y_{3}\right)\right), \ldots\right)_{0 \leq i \leq N}
$$

are projective coordinates of $\phi\left(s_{1} y_{1}+s_{2} y_{2}+s_{3} y_{3}\right)$.

Then if $\phi\left(s_{0} y_{0}\right)=\lambda_{0} \xi_{0}$ and $\phi\left(y_{i}\right)=\lambda_{i} \xi_{i}$ with $\xi_{i} \in \mathcal{O}_{K}^{N+1}(0 \leq i \leq 3)$, we have

$$
\begin{aligned}
\Phi_{y}(z) & =\lambda_{0}^{c_{9} D} P\left(1, z+y, A\left(A^{\prime}\left(\phi(z), \xi_{0}\right), F\left(\xi_{1}, \xi_{2}, \xi_{3}\right)\right)\right) \\
& =\lambda \sum_{(j, i) \in \mathcal{S}} p_{j, i} a_{j, i, y}(1, z, \phi(z)),
\end{aligned}
$$

where $\lambda \neq 0$; each $a_{j, i, y} \in K\left[\xi_{0}, \xi_{1}, \xi_{2}, \xi_{3}\right][Y, X]$ has bidegree at most $\left(c_{10} L, c_{11} D\right)$ with coefficients of deg $\leq c_{12} D S^{2}, \log \mathrm{ht} \leq c_{13}\left(D S_{0}^{2}+L \log S_{0}\right)$.

Choose $\phi_{i}(z)$ such that $\phi_{i}(0) \neq 0$. Then

$$
\begin{aligned}
\left(\frac{d}{d z}\right)^{t}\left[\frac{\Phi_{y}(z)}{\phi_{i}^{c_{11} D}(z)}\right] & =\lambda\left(\frac{d}{d z}\right)^{t} \sum_{(j, i) \in \mathcal{S}} p_{j, i} a_{j, i, y}\left(1, z, \frac{\phi(z)}{\phi_{i}(z)}\right) \\
& =\lambda \sum_{(j, i) \in \mathcal{S}} p_{j, i} a_{j, i, y}^{(t)}\left(1, z, \frac{\phi(z)}{\phi_{i}(z)}\right),
\end{aligned}
$$

with $a_{j, i, y}^{(t)} \in K\left[\xi_{0}, \xi_{1}, \xi_{2}, \xi_{3}\right][Y, X]$ of bidegree at most $\left(c_{10} L, c_{11} D+c_{14} t\right)$ with coefficient polynomials of

$$
\operatorname{deg} \leq c_{15} D S^{2} \text { and } \log h t \leq c_{16}\left(D S_{0}^{2}+L \log S_{0}+t \log (t+1)\right) .
$$

The system of equations

$$
\sum_{(j, i) \in \mathcal{S}} p_{j, i} a_{j, i, y}^{(t)}\left(1,0, \frac{\phi(0)}{\phi_{i}(0)}\right)=0 \quad(t=0, \ldots, T-1 ; y \in Y(S))
$$

has a nonzero solution by the box principle, provided $c^{\prime}$ is sufficiently small. Moreover, each $p_{j, i} \in \mathcal{O}_{K}$ has deg $\leq c_{17} D S^{2}$ and $\log$ ht $\leq c_{18} D S_{0}^{2}$.

Schwarz's lemma [9, Lemma 7.1.3] applied to circles of radii $r>\max _{y \in Y(S)}|y|$ and $R=r^{3 / 2}$ implies that

$$
\underset{|z| \leq r}{\log \max }\left|\Phi^{(t)}(z)\right| \leq c_{19} t \log t+c_{20} D R^{2}-c_{21} T S_{0} S^{3} \log \left(\frac{R}{r}\right) .
$$

Hence (4) holds when $r \leq c_{4} D$ and $t \leq c_{5} D^{3}$. This completes the proof of the lemma.

The estimates for $p_{1}, p_{2}$, and $p_{3}$ given by Lemma 3, applied with Lemma 2 , imply that for some $y_{*} \in Y(S)$ the exact order of vanishing, $t_{*}$, of $P(Y, X)$ at $y_{*}$ along $\phi$ satisfies $t_{*} \leq c_{22} D^{2} \log { }^{-1 / 3} D$. Hence

$$
\Phi^{\left(t_{*}\right)}\left(y_{*}\right) \neq 0 \text {. }
$$

There exist differential operators

$$
\Delta_{i}: K\left[Y, \frac{X_{0}}{X_{i}}, \ldots, \frac{X_{N}}{X_{i}}\right] \rightarrow K\left[Y, \frac{X_{0}}{X_{i}}, \ldots, \frac{X_{N}}{X_{i}}\right]
$$

such that, for any $P \in K[Y, X]$, 


$$
\left(\Delta_{i} P\right)\left(1, z, \frac{\phi_{0}}{\phi_{i}}, \ldots, \frac{\phi_{N}}{\phi_{i}}\right)=\left(\frac{d}{d z}\right) P\left(1, z, \frac{\phi_{0}}{\phi_{i}}, \ldots, \frac{\phi_{N}}{\phi_{i}}\right)
$$

on $\phi^{-1}\left(V_{i}\right)$, where $V_{i}=A \cap\left\{X_{i} \neq 0\right\}$. Let $\phi_{i_{*}}(z)$ denote the component function for $\phi(z)$ for which $\left|\phi_{i}\left(y_{*}\right)\right|$ is maximal. By the theory of theta functions,

$$
\left|\phi_{i_{*}}\left(y_{*}\right)\right| \geq \exp \left(-c_{23}\left(\left|y_{*}\right|^{2}+1\right)\right) \text {. }
$$

If we then choose addition laws $A_{0}, \ldots, A_{N}$ valid in a neighborhood of $y_{*}$ and put

$$
\Psi(z)=\frac{P\left(1, z+y_{*}, \phi\left(z+y_{*}\right)\right)}{\phi_{i_{*}}^{D}\left(z+y_{*}\right)}=\frac{P\left(1, z+y_{*}, A\left(\phi(z) \phi\left(y_{*}\right)\right)\right)}{A_{i_{*}}^{D}\left(\phi(z), \phi\left(y_{*}\right)\right)},
$$

we deduce from (6) that

$$
\left.\left(\frac{d}{d z}\right)^{t_{*}} \Psi(z)\right|_{z=0} \neq 0
$$

By homogeneity,

$$
\begin{aligned}
A_{i_{*}}^{D}\left(\frac{\phi(z)}{\phi_{i_{*}}(z)}, \frac{\phi\left(y_{*}\right)}{\phi_{i_{*}}\left(y_{*}\right)}\right) \Psi(z) & =P\left(1, z+y_{*}, A\left(\frac{\phi(z)}{\phi_{i_{*}}(z)}, \frac{\phi\left(y_{*}\right)}{\phi_{i_{*}}\left(y_{*}\right)}\right)\right) \\
& =P^{(1)}\left(1, z+y_{*}, \frac{\phi(z)}{\phi_{i_{*}}(z)}, \frac{\phi\left(y_{*}\right)}{\phi_{i_{*}}\left(y_{*}\right)}\right),
\end{aligned}
$$

where $P^{(1)} \in K\left[Y, X, X^{\prime}\right]$ is trihomogeneous of multidegree at most

$$
\left(c_{24} D^{2} \log { }^{-1 / 2} D, c_{25} D, c_{26} D\right)
$$

with coefficients in $K$ having deg $\leq c_{27} D^{2} \log { }^{-1 / 3} D, \log$ ht $\leq c_{28} D^{2} \log ^{2 / 3} D$. Then

$$
\begin{aligned}
{\left[A_{i_{*}}^{D}\left(\frac{\phi(z)}{\phi_{i_{*}}(z)}, \frac{\phi\left(y_{*}\right)}{\phi_{i_{*}}\left(y_{*}\right)}\right) \Psi^{\left(t_{*}\right)}(z)\right]_{z=0} } & =\left.\Delta_{i_{*}}^{t_{*}} P^{(1)}\left(Y, \frac{X}{X_{i_{*}}}, \frac{\phi\left(y_{*}\right)}{\phi_{i_{*}}\left(y_{*}\right)}\right)\right|_{\substack{X=\phi(0) \\
Y=\left(1, y_{*}\right)}} \\
& =P^{(2)}\left(1, y_{*}, \frac{\phi\left(y_{*}\right)}{\phi_{i_{*}}\left(y_{*}\right)}\right),
\end{aligned}
$$

with $P^{(2)} \in K[Y, X]$ of bidegree at most $\left(c_{29} D^{2} \log ^{-1 / 2} D, c_{30} D\right)$ and with coefficients having

$$
\begin{gathered}
\operatorname{deg} \leq c_{31}\left(D^{2} \log ^{-1 / 3} D+t_{*}\right) \leq c_{32} D^{2} \log { }^{-1 / 3} D, \\
\log \mathrm{ht} \leq c_{33}\left(D^{2} \log ^{2 / 3} D+t_{*} \log \left(t_{*}+1\right)\right) \leq c_{34} D^{2} \log ^{2 / 3} D,
\end{gathered}
$$

since $t_{*} \leq c_{35} D^{2} \log ^{-1 / 3} D$.

In addition, $P^{(2)}\left(1, y_{*}, \phi\left(y_{*}\right)\right) \neq 0$ and

$$
\log \left|P^{(2)}\left(1, y_{*}, \phi\left(y_{*}\right)\right)\right| \leq-c_{36} D^{4} \log ^{1 / 2} D \text {. }
$$

Since

$$
\frac{\left(\phi_{0}\left(y_{*}\right), \ldots, \phi_{N}\left(y_{*}\right)\right)}{\phi_{i_{*}}\left(y_{*}\right)}=\frac{\left(F_{0}\left(\lambda_{0} \xi_{0}, \lambda_{1} \xi_{1}, \lambda_{2} \xi_{2}, \lambda_{3} \xi_{3}\right), \ldots, F_{N}\left(\lambda_{0} \xi_{0}, \ldots, \lambda_{3} \xi_{3}\right)\right.}{F_{i_{*}}\left(\lambda_{0} \xi_{0}, \ldots, \lambda_{3} \xi_{3}\right)},
$$

we have 


$$
\begin{aligned}
\left|P^{(2)}\left(1, y_{*}, F\left(\lambda_{0} \xi_{0}, \ldots, \lambda_{3} \xi_{3}\right)\right)\right| & \left(\frac{\left|F_{i_{*}}\left(\lambda_{0} \xi_{0}, \ldots, \lambda_{3} \xi_{3}\right)\right|}{\left|\phi_{i_{*}}\left(y_{*}\right)\right|}\right)^{\operatorname{deg}_{X} P^{(2)}} \cdot\left|P^{(2)}\left(1, y_{*}, \phi\left(y_{*}\right)\right)\right|
\end{aligned}
$$

Therefore,

$$
\log \left|P^{(2)}\left(1, y_{*}, F\left(\lambda_{0} \xi_{0}, \ldots, \lambda_{3} \xi_{3}\right)\right)\right| \leq-c_{37} D^{4} \log { }^{1 / 2} D .
$$

Replacing $y_{*}$ and $\xi_{i}(i=0, \ldots, 3)$ by their integral polynomial representations in $\theta$ and $\theta_{1}$, by expanding out and regrouping terms we obtain a nonzero polynomial $P^{(3)}(x, y) \in K[x, y]$ with

$$
P^{(2)}\left(1, y_{*}, F\left(\lambda_{0} \xi_{0}, \ldots, \lambda_{3} \xi_{3}\right)\right)=\lambda_{4}^{c_{38} D} P^{(3)}\left(\theta, \theta_{1}\right) .
$$

Moreover, $P^{(3)}(x, y)$ satisfies

$$
\operatorname{deg}_{x} P^{(3)} \leq c_{39} D^{2} \log ^{-1 / 3} D, \quad \operatorname{deg}_{y} P^{(3)} \leq n
$$

with coefficients in $K$ having

$$
\operatorname{deg} \leq c_{40} D^{2} \log { }^{-1 / 3} D, \quad \log \mathrm{ht} \leq c_{41} D^{2} \log ^{2 / 3} D,
$$

such that

$$
\log \left|P^{(3)}\left(\theta, \theta_{1}\right)\right| \leq-c_{42} D^{4} \log { }^{1 / 2} D .
$$

From the estimates for the coefficients of $P^{(3)}$ given above, there exists a polynomial $\delta(x, y) \in \mathbf{Z}[x, y]$ with $\delta\left(\theta, \theta_{1}\right) \neq 0$ such that

$$
P^{(4)}(x, y)=\delta(x, y) P^{(3)}(x, y)
$$

has coefficients in $\mathcal{O}_{K}$ and satisfies the same estimates as $P^{(3)}$, possibly with different constants.

Put

$$
Q_{D}(x)=N_{K / \mathbf{Q}^{(\theta)}}\left(P^{(4)}\left(x, \theta_{1}\right)\right) .
$$

Then $Q_{D} \in \mathbf{Z}[x]$ is a nonzero polynomial with $\operatorname{deg} Q_{D} \leq c_{43} D^{2} \log ^{-1 / 3} D$,

$$
\log \text { ht } Q_{D} \leq c_{44} D^{2} \log ^{2 / 3} D, \text { and } \log \left|Q_{D}(\theta)\right| \leq-c_{45} D^{4} \log ^{1 / 2} D \text {. }
$$

For each choice of $D \geq D_{2}$,

$$
\frac{(D+1)^{2}(\log (D+1))^{-1 / 3}}{D^{2}(\log D)^{-1 / 3}} \leq a, \quad \frac{(D+1)^{2}(\log (D+1))^{2 / 3}}{D^{2}(\log D)^{2 / 3}} \leq a,
$$

and

$$
(\log D)^{1 / 6}>c_{46}(2 a+1)
$$

hold with $a \geq 5 / 2$. So for $t \in \mathbf{N}$ put

$$
\begin{aligned}
P_{t}(X) & =Q_{D_{2}+t}(X), \\
\delta_{t} & =c_{43}\left(D_{2}+t\right)^{2}\left(\log \left(D_{2}+t\right)\right)^{-1 / 3}, \\
\gamma_{t} & =c_{44}\left(D_{2}+t\right)^{2}\left(\log \left(D_{2}+t\right)\right)^{2 / 3} .
\end{aligned}
$$


Then, for $t \in \mathbf{N}$,

$$
\log \left|P_{t}(\theta)\right|<-(2 a+1) \delta_{t}\left(\delta_{t}+\gamma_{t}\right),
$$

where deg $P_{t} \leq \delta_{t}$ and $\log$ ht $P_{t} \leq \gamma_{t}$. Lemma 1 implies that $P_{t}(\theta)=0$ for each $t$, contradicting the transcendence of $\theta$. This completes the proof of the Theorem in case $(b)$.

IV. For case (a) the same general proof applies. If we assume that the hypotheses of the Theorem in case (a) hold but that $\overline{\phi(\mathbf{C})}$ is not an elliptic curve, we obtain the following analogue to Lemma 4 . Here $\mathcal{G}$ defines $A$ in $\mathbf{P}_{N}$.

LEMMA 5. With $Y, \phi$, and $A$ as above there exists $D_{1} \geq 0$ such that for each integer $D \geq D_{1}$ there exists a homogeneous polynomial $P(X) \in \mathcal{O}_{K}[X] \backslash \mathcal{S}$ of degree at most $c_{47} D$, with coefficients satisfying

$$
\operatorname{deg} \leq c_{48} D^{7 / 5} \log ^{2 / 5} D, \quad \log \mathrm{ht} \leq c_{49} D^{7 / 5} \log ^{2 / 5} D
$$

such that $\Phi(z)=P\left(\phi_{0}(z), \ldots, \phi_{N}(z)\right)$ satisfies

$$
\log _{|z|=r} \max \left|\Phi^{(t)}(z)\right| \leq-c_{50} D^{2} r \log D,
$$

provided $0<r<c_{51} D^{5 / 6}, 0 \leq t<c_{52} D^{2}$.

The proof depends on the choice of parameters

$$
T=\llbracket c^{\prime} D^{7 / 5} \log { }^{-3 / 5} D \rrbracket, \quad S=\llbracket D^{1 / 5} \log ^{1 / 5} D \rrbracket
$$

for $0<c^{\prime}<1$ sufficiently small. The inequality (7) is deduced from the observation that for some $n \in \mathbf{Z}, n \cdot y_{0} \neq 0$ lies in the kernel of $\phi$. Therefore for each $i$ the function $F_{i}(z)=\phi_{i}^{-D}(z) \Phi(z)$ is periodic.

With $G=A$ and $\Gamma=\phi(Y)$ the estimates $p_{1}(\Gamma, G) \geq 2$ and $p_{2}(\Gamma, G)=3$, applied with Lemma 2, yield $y_{*} \in Y(S)$ (here $S=(S, S, S, S)$ ) such that, for some $t_{*} \leq$ $c_{53} T, \Phi^{\left(t_{*}\right)}\left(y_{*}\right) \neq 0$. The conclusion of the proof is as before.

Note added in proof. Masser and Wüstholz [11] have obtained several general results concerning the algebraic independence of values of elliptic functions. In particular, Theorem 5 of their paper is our Corollary 1 with the stronger hypothesis that all of $\mathcal{P}\left(u_{1} v_{1}\right), \ldots, \mathcal{P}\left(u_{1} v_{4}\right)$ are algebraic.

We also note that in Corollary 1 the hypothesis that all of the values $\mathcal{P}\left(u_{i} v_{j}\right)$ are finite may be dropped, if we alter the conclusion to say that at least two of the finite values among $u_{i}, v_{j}, \mathcal{P}\left(u_{i} v_{j}\right)(1 \leq i \leq 2,1 \leq j \leq 4)$ are algebraically independent. Corollary 2 would then be that for any nonzero $u \in \mathbf{C}$, at least one of $\mathcal{P}(u), \ldots, \mathcal{P}\left(u^{5}\right)$ is transcendental.

\section{REFERENCES}

1. D. Bertrand, Problèmes locaux, Appendice I, Nombres transcendants et groupes algébriques by M. Waldschmidt, Astérisque, 69-70, Soc. Math. France, Paris, 1979.

2. W. D. Brownawell, Sequences of Diophantine approximations, J. Number Theory 6 (1974), 11-21. 
3. - The algebraic independence of certain numbers related by the exponential function, J. Number Theory 6 (1974), 22-31.

4. W. D. Brownawell and K. K. Kubota, The algebraic independence of Weierstrass functions and some related numbers, Acta Arith. 33 (1977), 111-149.

5. D. W. Masser and G. Wüstholz, Zero estimates on group varieties I, Invent. Math. 64 (1981), 489-516.

6. - Zero estimates on group varieties II, Invent. Math. 80 (1985), 233-267.

7. J.-P. Serre, Quelques propriétés des groupes algébriques commutatifs. Nombres transcendants et groupes algébriques by M. Waldschmidt, Astérisque, 69-70, Soc. Math. France, Paris, 1979.

8. M. Waldschmidt, Nombres transcendants, Lecture Notes in Math., 402, Springer, Berlin, 1974.

9. - Nombres transcendants et groupes algébriques, Astérisque, 69-70, Soc. Math. France, Paris, 1979.

10. _ Solution du huitième probleme de Schneider, J. Number Theory 5 (1973), 191-202.

11. D. W. Masser and G. Wüstholz, Algebraic independence of values of elliptic functions, Math. Ann. 276 (1986), 1-17.

School of Mathematics

Institute for Advanced Study

Princeton, NJ

and

Department of Mathematics

University of Colorado

Boulder, CO 80309 